\title{
An Effective Non-Invasive Joint Distraction Technique for Ankle Arthroscopy
}

\section{Keywords: Ankle joint; Arthroscopy; Traction; Distraction Abstract \\ Both invasive and non-invasive methods of ankle joint distraction for arthroscopic procedures have been described. Challenges prevail in achieving an adequate joint distraction for instrumentation inside the ankle joint especially in patients of large stature. We describe a technique, which safely offers effective joint distraction during ankle arthroscopy without the need for resorting to traction pin.}

\section{Introduction}

Ankle joint arthroscopy is a commonly performed procedure for a variety of indications including: impingement lesions, osteochondral defects, arthroscopy assisted arthrodesis and excision of loose bodies. Over the last three decades this procedure has evolved from a diagnostic to a therapeutic intervention. With the inherent difficulty in arthroscopic visualisation of the ankle, the tibiotalar joint space if often too narrow to insert both scope and instruments [1]. Both invasive and non-invasive methods of ankle joint distraction for arthroscopic procedures have been described. Previous authors have described the use of long bone fracture distractors; others recommend

\section{Journal of \\ Orthopedics \& Rheumatology}

\section{Tarek Boutefnouchet ${ }^{1,2 *}$, Thomas Laios ${ }^{2}$, Peyman Bakhshayesh ${ }^{1}$, Ashgar S Ali ${ }^{1}$}

${ }^{I}$ Department of Trauma and Orthopaedics, Queen Elizabeth Hospital, Birmingham B15 2WB, UK

${ }^{2}$ Department of Trauma and Orthopaedic Surgery, Heart of England NHS Foundation Trust, Bordesley Green East, Birmingham B9 $5 S S, U K$

*Address for Correspondence

Tarek Boutefnouchet, MBChB, MRCS, PGCert ME, Department of Trauma and Orthopaedics, Queen Elizabeth Hospital, Mindelsohn Way, Birmingham B15 2WB, UK, E-mail: tboutefnouchet@hotmail.com

Submission: 29 September, 2016

Accepted: 10 November, 2016

Published: 16 November, 2016

Copyright: ๑ 2016 Boutefnouchet T, et al. This is an open access article distributed under the Creative Commons Attribution License, which permits unrestricted use, distribution, and reproduction in any medium, provided the original work is properly cited.

the use of bandages or specially designed foot sling with varying advantages and limitations [2,3]. These methods have been in use for a long period of time, nevertheless achieving a safe and effective joint distraction especially in the patients of large stature remains a challenge $[4,5]$. To the authors knowledge there are no description of ankle arthroscopy distraction technique using a standard fracture table. A comprehensive description of all tibiotalar distraction

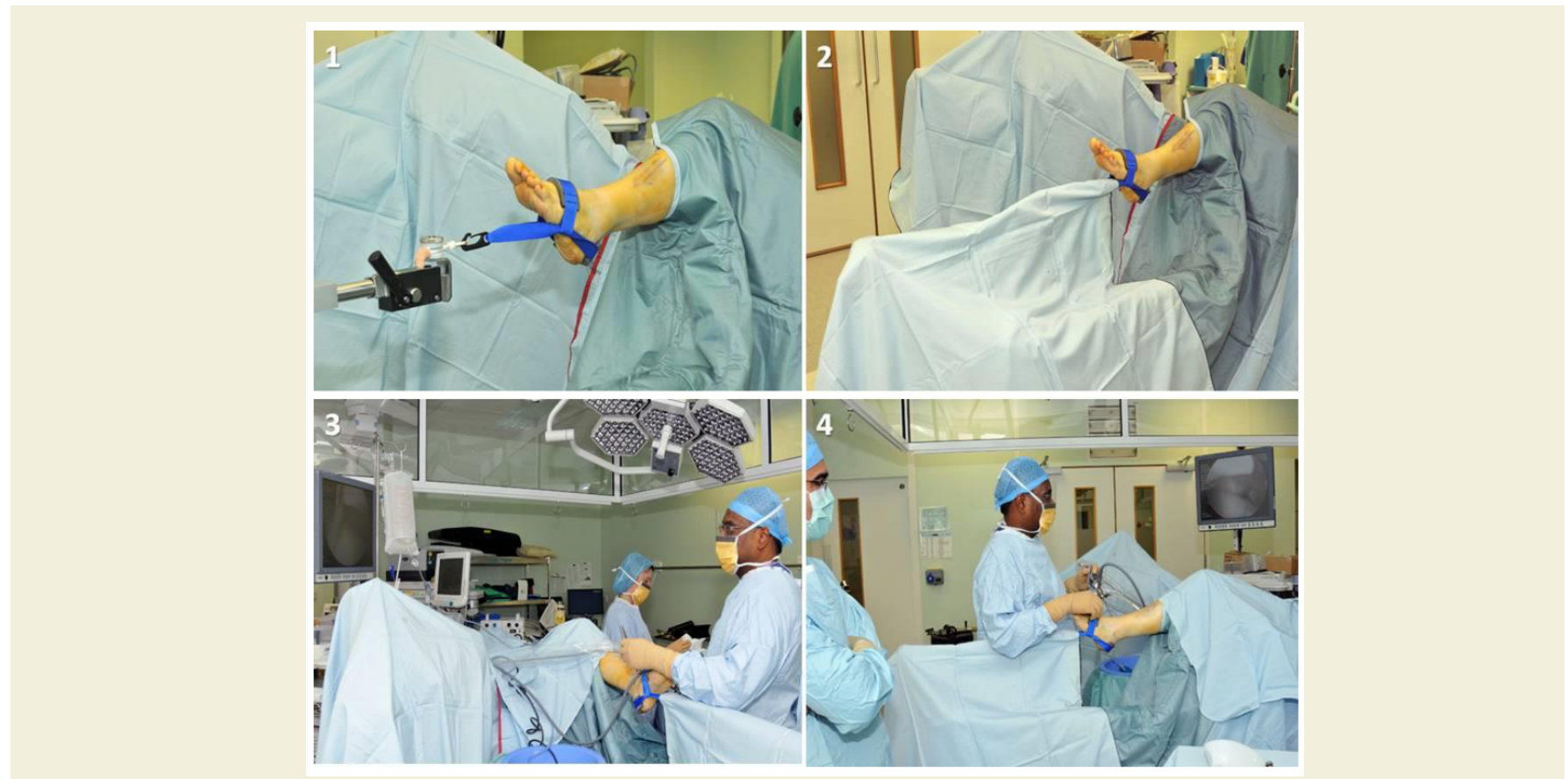

Figure 1: (1) With the patient supine the foot is placed into foot strap and attached to the traction table mechanism. (2) The non-operated leg is elevated and standard draping applied. (3) The stack is place facing the surgeon. (4) Position of both leg and traction allows the surgeon ease of access to both medial and lateral sides while maintaining a good view of the screen. 
Citation: Boutefnouchet T, Laios T, Baksheysh P, Ali AS. An Effective Non-Invasive Joint Distraction Technique for Ankle Arthroscopy. J Orthopedics Rheumatol. 2016; 3(2): 2.

ISSN: $2334-2846$

techniques was beyond the scope of present article. In addition, Palladino described these methods in the past and the literature has not had any new variation in the available techniques [6]. We describe a non-invasive technique which safely offers effective joint distraction during ankle arthroscopy.

\section{Technique}

Prior to the procedure patients receive a general or regional anaesthetic in the usual manner. A proximal thigh tourniquet is placed on the operated side and the lower limb draped below the knee level as shown on the figure. Patient is positioned supine on a standard orthopaedic traction table. The posterior aspect of the leg is supported proximally to provide counter traction; the foot is then placed into an Acufex ${ }^{\text {tw }}$ ankle distraction foot strap (Smith \& Nephew Inc, Andover, MA 01810, USA) and connected to the standard traction mechanism (Figure 1(1)). The contralateral leg is elevated in the lithotomy position and standard draping is applied to both legs (Figure 1(2)). The amount of traction and foot position can be easily adjusted as required. This technique allows ease of access to both medial and lateral portals (Figures 1(3) and 1(4)) both surgeon and assistant remain free. The extent of traction is easily controlled by the surgeon or assistant through the drape using the distraction control handles, equally the position of the foot and ankle can be easily altered within the foot strap. The posterior distal aspect of the thigh is positioned on a very well padded leg support taking care to avoid direct compression of the popliteal fossa.

\section{Discussion}

The exact method of joint distraction is determined by the surgeon and joint pathology, some indications require more distraction than others e.g. talar dome lesion, meniscoid lesion, gutter pathology, and adhesive capsulitis. This article presents one of many options available to the arthroscopic surgeon. Beals et al. described the use of traction pin for distraction during prone posterior ankle arthroscopy [3]. These authors reported safe and effective arthroscopic instrumentation with this technique. Nevertheless, these are achieved with used of an invasive method with traction pin. The latter remains an additional procedure with additional operative risks. In contrast other authors have advocated changing the position of portals to aid in visualisation depending on the technique and body habitus [7] Posterior ankle portals combined with non-invasive distraction have found less popularity due to the technical challenges and the potential risk of neurovascular injuries $[8,9]$.

In a recent study, Barg et al. evaluated the extent of talar dome visibility comparing matched pairs of specimen using either noninvasive strap distraction or calcaneal traction pin [8]. For anterior ankle arthroscopy the quantitative comparison showed that both techniques achieved comparable mean talar dome visualisation [8]. In terms of clinical translation of this work, non-invasive strap distraction should therefore suffice in most clinical indications.
Access to the posterior aspect of the talar dome might however require variation in the technique with the adjunct of calcaneal traction and posterior portals.

Our technique aims to provide optimal field of vision and manoeuvring room for instruments without the need for invasive distraction which carries the risk of pin site infection, neurovascular injury and fracture. Our technique was found to be particularly useful in patients of large stature, including military personnel patients commonly treated in our centre. Hence, it offers a safer alternative to invasive ankle traction when more sustained joint distraction is required. Allowing the ankle to dorsiflex and plantarflex, visualisation of the tibiotalar joint space can be achieved and adjusted according to the need of the procedure. We find this easily achievable and the traction table attachment mechanism gives a stable and very well controlled movements hence allowing for gross and fine traction to be applied. If there is need to alter the height of the leg we recommend that a non-scrubbed member of the theatre team adjusts the lower control handles on the traction bar. Distraction force and duration is closely monitored and controlled to minimize the risk of traction or compression nerve injuries. In a series of heterogeneous clinical cases carried out by the senior author there were no reports of neurovascular injuries. There was no incidence of transient nerve damage due to the straps. Comparable non-invasive distractions do not report proximal nerve injuries [9]. The degree of control provided by this mechanism helps reduce the risk of neurovascular injuries since less strenuous efforts are required to maintain visualisation.

\section{References}

1. van Dijk CN, van Bergen CJ (2008) Advancements in ankle arthroscopy. J Am Acad Orthop Surg 16: 635-646.

2. Miyamoto W, Takao M, Komatu F, Uchio Y (2008) Technique tip: the bandage distraction technique for arthroscopic arthrodesis of the ankle joint. Foot Ankle Int 29: 251-253.

3. Beals TC, Junko JT, Amendola A, Nickisch F, Saltzman CL (2010) Minimally invasive distraction technique for prone posterior ankle and subtalar arthroscopy. Foot Ankle Int 31: 316-319.

4. Casteleyn PP, Handelberg F (1995) Distraction for ankle arthroscopy. Arthroscopy 11: 33-34.

5. Corso SJ, Zimmer TJ (1995) Technique and clinical evaluation of arthroscopic ankle arthrodesis. Arthroscopy 11: 585-590.

6. Palladino SJ (1994) Distraction systems for ankle arthroscopy. Clin Podiatr Med Surg 11: 499-511.

7. Frey C, Gasser S, Feder K (1994) Arthroscopy of the subtalar joint. Foot Ankle Int 15: 424-428.

8. Barg A, Saltzman CL, Beals TC, Bachus KN, Blackenhorn BD, et al. (2016) Arthroscopic talar dome access using a standard versus wire-based traction method for ankle joint distraction. Arthroscopy 32: 1367-1374.

9. Young BH, Flannigan RM, DiGiovanni BF (2011) Complications of ankle arthroscopy utilizing a contemporary non-invasive distraction technique. J Bone Joint Surg Am 93: 963-968. 